\title{
Optimal Control of Stochastic Dynamic Systems of a Random Structure with Poisson Switches and Markov Switching
}

\author{
Svitlana V. Antonyuk (i), ${ }^{1}$ Marian F. Byrka $\mathbb{D}^{2},{ }^{2}$ Mykola Y. Gorbatenko $\left(\mathbb{D},{ }^{3}\right.$ \\ Taras O. Lukashiv $\mathbb{D}^{3}{ }^{3}$ and Igor V. Malyk $\mathbb{D}^{1}$ \\ ${ }^{1}$ Institute of Physical Engineering and Computer Science, Yuriy Fedkovych Chernivtsi National University, 14 Rivnenska Street, \\ Chernivtsi 58000, Ukraine \\ ${ }^{2}$ Department of Pedagogy, Psychology and Education Management, \\ Institute of Postgraduate Pedagogical Education of Chernivtsi Region, Chernivtsy 58000, Ukraine \\ ${ }^{3}$ Department of Mathematics and Informatics, Yuriy Fedkovych Chernivtsi National University, 28 Universytetska Street, \\ Chernivtsi 58012, Ukraine
}

Correspondence should be addressed to Taras O. Lukashiv; t.lukashiv@gmail.com

Received 19 March 2020; Revised 25 May 2020; Accepted 30 May 2020; Published 24 June 2020

Academic Editor: Yongqiang Fu

Copyright (c) 2020 Svitlana V. Antonyuk et al. This is an open access article distributed under the Creative Commons Attribution License, which permits unrestricted use, distribution, and reproduction in any medium, provided the original work is properly cited.

The problem of synthesis of the optimal control for a stochastic dynamic system of a random structure with Poisson perturbations and Markov switching is solved. To determine the corresponding functions for Bellman functional and optimal control the system of ordinary differential equation is investigated.

\section{Introduction}

Systems with Markov parameters belong to an important class of systems describing processes of the rapid changes in states that occur in many cases, for example, in industry, in queuing systems [1], in ecological systems [2], in economics and finance, and in modeling of a microgrid [3]. These systems are mathematical models of the hybrid dynamic systems, in which one part of the variables changes continuously and the other part changes discretely. The most used hybrid models are models described by differential equations. This article focuses on this area of research.

Let us pay attention to the fact [4] that such abrupt changes in the systems are discrete events and are assumed to be modelled by a Markov chain taking values in a finite value set. Practical motivations as well as many theoretical results for the Markovian jump system can be found, for example, in [5-7]. [4]:

$$
\begin{aligned}
\dot{x}(t)= & (A(\xi(t))+\Delta A(\xi(t)) x(t))+(B(\xi(t)) \\
& +\Delta B(\xi(t)) u(t)), \quad t \geq 0, \\
x(0)= & x_{0}, \\
\xi(0)= & \xi_{0} .
\end{aligned}
$$

Here, $x \in \mathbf{R}^{n}$ is a state vector, $\xi$ is the Markov chain with finite number of states, $A, B$ are defined constant matrices for each fixed $\xi, \Delta A, \Delta B$ are indefinite matrices that satisfy the specific conditions described in the following, and $u \in \mathbf{R}^{m}$ is a control. In this case, the problem of the synthesis of optimal control with constant inverse communication for such systems is solved.

In [2], the optimal control problem for the Ito linear stochastic system with the uncertainty of the following form is solved:

$$
\begin{aligned}
\dot{x}(t)= & (A(\xi(t))+\Delta A(\xi(t)) x(t))+(B(\xi(t)) \\
& +\Delta B(\xi(t)) u(t))+C(\xi(t)) x(t) w(t), \quad t \geq 0, \\
x(0)= & x_{0}, \\
\xi(0)= & \xi_{0} .
\end{aligned}
$$


Here and in the following, $w(t), t \geq 0$, is a Wiener process.

In [6], the optimal control problem for the Ito linear stochastic system of the following form is solved:

$$
\begin{aligned}
\dot{x}(t)= & A_{0}(t, \xi(t)) x(t)+B_{0}(t, \xi(t)) u(t) \\
& +\sum_{k=1}^{r}\left[A_{k}(t, \xi(t)) x(t)+B_{k}(t, \xi(t)) u(t)\right] w_{k}(t) .
\end{aligned}
$$

The nonlinear case of the Ito stochastic system is considered in [4]. In this paper, the sufficient optimality conditions are obtained in the case of an infinite horizon for the system

$$
\dot{x}(t)=\left(f(x, \xi(t))+g_{1}(x, \xi(t), u(t))\right) \mathrm{d} t+g_{2}(x, \xi(t)) w(t) .
$$

In [7], optimality conditions were obtained in the case of an infinite horizon for a linear stochastic system with finite aftereffect and Markov parameters:

$$
\begin{aligned}
\dot{x}(t)= & A_{0}(\xi(t)) x(t)+A_{1}(\xi(t)) x(t-h(t)) \\
& +B_{0}(\xi(t)) u(t)+B_{1}(\xi(t)) w(t) \\
& +g(x(t), x(t-h(t)), u(t), w(t), \xi(t)) .
\end{aligned}
$$

A great contribution to the development of systems with Markov parameters was introduced by Katz [8]. In this work, a Markov process can be either a Markov chain or a continuous Markov process. In addition, it is advisable to consider the case of perturbations of the impulse type, this is when, for example, the moments of time in which the discontinuities of the phase trajectories of the process are possible, and they are known in advance. For deterministic and difference systems, this situation was studied in detail in [9].

Stochastic systems of random structure according to Katz [8] with impulse Markov perturbations according to Tsarkov were considered in [10-13]. Stability in different probabilistic senses has been investigated, and the problem of optimal stabilization, solution of which is the control stabilizing the system to stochastically stable, is solved. In these papers, the absence of perturbation points was assumed, but in [14-16], the existence and uniqueness of the solution of a system of differential-difference equations with Markov parameters and switching in the presence of perturbation points were proved. Therefore, we can consider the problem of stability and optimal control for such systems.

Furthermore, in the theory of control of classical problems, there are problems of constructing the control that minimizes a certain quality functional. This problem is wider than the problem of optimal stabilization because it does not include stabilization of the solution $x(\mathrm{t})$. The monograph [17] is one of the main papers in which the general theory of controllable processes and the theory of controlled stochastic differential equations are presented. Here, the classical problems of optimal stopping of random processes are considered, a rigorous proof of the derivation of Bellman's equations [18] is presented, and applications of
Bellman's equations for constructing optimal controls are considered.

Controllable systems with finite aftereffect of the deterministic and stochastic type are considered in [19]. The paper [20] is devoted to the synthesis of optimal control of linear stochastic dynamic systems with finite aftereffect and Poisson perturbations. The general form of the Bellman equation and the Bellman functional for a stochastic dynamical system of a random structure with Markov switching was obtained in [21], which is a sufficient condition for the existence of optimal control, and the problem of synthesis of optimal control for such systems was solved in [22].

The switching times of Markov processes in [21, 22] are assumed to be known; however, in many cases, abrupt changes in the trajectories of the process at random moments are also possible. Such situations are adequately described by taking into account the term in the equation of motion, which is the integral of the Poisson measure. Stochastic systems with semi-Markov switching were introduced in [23]. In this paper, the conditions of weak convergence of semi-Markov random evolutions to the diffusion process are considered. Also, sufficient conditions for the stability of prelimited processes are discussed. In the articles $[15,16]$, Tsarkov et al. discussed the influence of Markov perturbation on the solutions and applied stochastic differential equations for applied problems.

This paper is devoted to the synthesis of optimal control of stochastic dynamic systems of a random structure that are under the influence of impulse Markov switching at known moments of time with allowance for Poisson perturbations that allow describing discontinuities of trajectories at random moments.

\section{Main Result}

Consider the stochastic random structure system given by the Ito stochastic differential equation

$$
\begin{array}{r}
\mathrm{d} x(t)=a(t, \xi(t), x(t), u(t)) \mathrm{d} t+b(t, \xi(t), x(t), u(t)) \mathrm{d} w(t) \\
\quad+\int_{\mathbf{R}^{m}} c(t, \xi(t), x(t), u, z) \widetilde{v}(\mathrm{~d} z, \mathrm{~d} t), \quad t \in \mathbf{R}_{+} \backslash K,
\end{array}
$$

with Markov switching

$$
\begin{aligned}
\left.\Delta x(t)\right|_{t=t_{k}} & =g\left(t_{k}-, \xi\left(t_{k}-\right), \eta_{k}, x\left(t_{k}-\right)\right) \\
t_{k} \in K & =\left\{t_{n} \Uparrow\right\}, \\
\lim _{n \longrightarrow \infty} t_{n} & =+\infty,
\end{aligned}
$$

and initial conditions

$$
\begin{gathered}
x\left(t_{0}\right)=x_{0} \in \mathbf{R}^{m}, \\
\xi\left(t_{0}\right)=y \in \mathbf{Y}, \\
\eta_{k_{0}}=h \in \mathbf{H} .
\end{gathered}
$$

Here, $\xi(t)$ is the continuous Markov process with values in the space $\mathbf{Y}:=\left\{y_{1}, \ldots, y_{N}\right\},\left\{\eta_{k}, k \geq 0\right\}$ is the Markov 
chain with values in space $\mathbf{H} ; x:[0,+\infty) \times \Omega \longrightarrow \mathbf{R}^{m} ; w(t)$ is the $m$-measurable standard Wiener process; $\widetilde{v}(\mathrm{~d} z, \mathrm{~d} t)=$ $v(\mathrm{~d} z, \mathrm{~d} t)-\mathbf{E} v(\mathrm{~d} z, \mathrm{~d} t), \mathbf{E} v(\mathrm{~d} z, \mathrm{~d} t)=\Pi(\mathrm{d} z) \mathrm{d} t$, is the centered Poisson measure $[24,25]$; and the processes $w, v, \xi$, and $\eta$ are independent $[24,25]$ and defined in the probability basic $\left(\Omega, F,\left\{F_{t}\right\}_{t \geq 0}, P\right)$.

The trajectories of process $x(t), t \geq 0$, belong to the Skorokhod space D [17], control $u(t):=u(t, x(t)):[0 ; T] \times$ $\mathbf{R}^{m} \longrightarrow \mathbf{R}^{m}$ is the $m$-measurable function from a class of admissible controls $U$ [19]; coefficients $a: \mathbf{R}_{+}$ $\times \mathbf{Y} \times \mathbf{R}^{m} \times \mathbf{R}^{m} \longrightarrow \mathbf{R}^{m}, b: \mathbf{R}_{+} \times \mathbf{Y} \times \mathbf{R}^{m} \times \mathbf{R}^{m} \longrightarrow \mathbf{R}^{m} \times \mathbf{R}^{m}$, and $c$ : $\mathbf{R}_{+} \times \mathbf{Y} \times \mathbf{R}^{m} \times \mathbf{R}^{m} \times \mathbf{R}^{m} \longrightarrow \mathbf{R}^{m}$ and function $g: \mathbf{R}_{+} \times \mathbf{Y} \times \mathbf{H} \times \mathbf{R}^{m} \longrightarrow \mathbf{R}^{m}$ are measurable functions which satisfy the conditions for the existence and uniqueness of a solution of problems (6)-(8). An example of the existence and uniqueness conditions is the Lipschitz condition and linear growth condition [17].
Consider a sequence of functions $v_{k}(t, x):\left[t_{0}, T\right] \times \mathbf{R}^{m} \longrightarrow \mathbf{R}^{1}, k \geq 0$, and define the class of the functions:

$$
V:=\left\{f(t, x): f \in C^{1,2}\left(\mathbf{R}_{+} \times \mathbf{R}^{m}\right)\right\} .
$$

The weak infinitesimal operator (WIO) $v_{k}(t, x) \in V[24]$ $\mathscr{L} v_{k}(t, x)=\lim _{\Delta \longrightarrow 0+} \frac{1}{\Delta}\left\{\mathbf{E}_{t, x} v_{k}\left(t+\Delta, x\left(t+\Delta, t_{k}, y, h, u\right)\right)-v_{k}(t, x)\right\}$,

is defined on the functionals $v_{k}(t, x) \in V$.

Here, $x(t)=x\left(t, t_{k}, y, h, u\right)$ is a strong solution of (6) on $t \in\left[t_{k}, t_{k+1}\right)$ by the control $u_{k} \in U$, which is constructed on the interval $\left[t_{k}, t_{k+1}\right)$. The optimal control problem is to find a control $u_{k}^{0}, k \geq 0$, from the class $U$ that minimizes the functional of quality [19]:

$$
I\left(u_{k}^{0}\right)=I^{u_{k}^{0}}(t, x)=\inf _{u \in U}\left(\sum_{k=0}^{N} \mathbf{E}_{t_{k}, x_{k}}\left\{F(x(T))+\int_{t}^{T} G(s, x(s), u(s)) \mathrm{d} s\right\}\right) .
$$

For some fixed $T>0$,

$F(x) \geq 0, G(t, x, u) \geq 0, \mathbf{E}_{t, x}\{f\}=\mathbf{E}\{f / x(t)=x\}$,

where

$N=\max \left\{k: t_{k}<T\right\}$.

It is necessary to prove some auxiliary properties in order to obtain sufficient conditions of optimality. Let us consider the main statements about properties of the infinitesimal operator.

\section{Lemma 1. Let}

(1) Unique solution of problems (6)-(8) exists with finite second moment for each $t$

(2) Sequence of functionals $v_{k}:[0, T] \times \mathbf{R}^{m} \longrightarrow \mathbf{R}^{1}, k \geq 0$, from class $V$ exists

(3) WIO $\mathrm{L}_{k}^{v}(s, x)$ be defined for $s \in\left[t_{k}, T\right]$ on solutions of (6) $-(8)$

Then, $\forall \widetilde{t}_{1}, \widetilde{t}_{2} \in\left[t_{0}, T\right]$ and the quality

$$
\mathbf{E}_{t_{k}, x_{k}} v_{k}\left(\widetilde{t}_{2}, x\left(\widetilde{t}_{2}\right)\right)-\mathbf{E}_{t_{k}, x_{k}} v_{k}\left(\widetilde{t}_{1}, x\left(\widetilde{t}_{1}\right)\right)=\int_{\tilde{t}_{1}}^{\tilde{t}_{2}} \mathbf{E}_{t_{k}, x_{k}} L v_{k}(s, x(s)) \mathrm{d} s,
$$

hold.

Proof. Then, the Markov process $x\left(t, t_{k}, y, h, u\right)$ with respect to the minimal $\sigma$-algebra $\mathrm{F} \sim \widetilde{t}_{1} \tilde{t}_{2}$ that is built on the interval $\left[\widetilde{t}_{1}, \tilde{t}_{2}\right], \tilde{t}_{1}, \tilde{t}_{2} \in\left[t_{0} ; T\right]$, satisfies the Dynkin formula [24]:

$$
\begin{aligned}
& \mathbf{E}_{t_{k}, x_{k}} v_{k}\left(\tilde{t}_{1}+\tau\left(\tilde{t}_{2}\right), x\left(\tilde{t}_{1}+\tau\left(\tilde{t}_{2}\right), t_{k}, y, h, u\right)\right) \\
& =v_{k}(t, x)+\mathbf{E}_{t_{k}, x_{k}} \int_{t_{0}}^{\tau_{\mathrm{r}}\left(\tilde{t}_{2}\right)} L v_{k}\left(\tilde{t}_{1}+\tau, x\left(\tilde{t}_{1}+\tau, t_{k}, y, h, u\right)\right) \mathrm{d} \tau,
\end{aligned}
$$

where $\tau_{r}=\inf _{t}\{|x(t)|>r\}$ and $\tau_{r}\left(\tilde{t}_{2}\right)=\min \left\{\tau_{r}, \tilde{t}_{2}\right\}$.
If $\lim _{r \rightarrow \infty} \tau_{r}\left(\widetilde{t}_{2}\right)=\widetilde{t}_{2}$, then for the solution of (6)-(8), we can obtain the equal

$$
\begin{aligned}
& \mathbf{E}_{t_{k}, x_{k}} v_{k}\left(\tilde{t}_{1}+\tilde{t}_{2}, x\left(\tilde{t}_{1}+\tilde{t}_{2}, t_{k}, y, h, u\right)\right) \\
& =v_{k}(t, x)+\mathbf{E}_{t_{k}, x_{k}} \int_{t_{0}}^{\tilde{t}_{2}} L v_{k}\left(\tilde{t}_{1}+\tau, x\left(\tilde{t}_{1}+\tau, t_{k}, y, h, u\right)\right) \mathrm{d} \tau .
\end{aligned}
$$

Analogously, we should write the Dynkin formula [24] on the interval $\left[t_{0}, \widetilde{t}_{1}\right]$, and subtracting it from (14), we obtain (12). Lemma 1 is proved.

Lemma 2. Let

(1) Conditions (1) and (2) of Lemma 1 be satisfied.

(2) For $\forall t \in\left[t_{0}, T\right], \forall v_{k} \in V, k \geq 0$, the equation

$$
L v_{k}(t, x)+G\left(t, x, u_{k}(t, x)\right)=0,
$$

with boundary condition

$$
v_{k}(T, x)=0, \quad k \geq 0,
$$

hold, where $L v_{k}(t, x)$ is the WIO defined by (10).

Then, $v_{k} \in V, k \geq 0$, can be given as

$$
v_{k}(t, x)=\mathbf{E}_{t_{k}, x_{k}}\left\{\int_{t}^{T} G(s, x, u(s, x)) \mathrm{d} s\right\}, \quad t \in\left[t_{k}, T\right] .
$$

Proof. Consider the solution $x(t) \in \mathbf{R}^{m}$ of (6)-(8) for $t \in\left[t_{k}, T\right]$, which is constructed according to the initial condition. 
Let us integrate (15) with respect to $t$ from $t_{k}$ to $T$ and calculate the expectation. We obtain

$$
\mathbf{E}_{t_{k}, x_{k}}\left\{\int_{t_{k}}^{T} L v_{k}(s, x) \mathrm{d} s\right\}+\mathbf{E}_{t_{k}, x_{k}}\left\{\int_{t_{k}}^{T} G(s, x) \mathrm{d} s\right\}=0 .
$$

According to Lemma 1, the first term in (18) exists, and it is equal to increment (12):

$$
\begin{aligned}
\mathbf{E}_{t_{k}, x_{k}} & \left\{\int_{t}^{T} L v_{k}(s, x(s)) \mathrm{d} s\right\} \\
= & \mathbf{E}_{t_{k}, x_{k}} v_{k}(T, x(T))-\mathbf{E}_{t_{k}, x_{k}} v_{k}(t, x(t)) \\
= & -\mathbf{E}_{t_{k}, x_{k}} v_{k}(t, x)=-v_{k}(t, x),
\end{aligned}
$$

where $\quad \mathbf{E}_{t_{k}, x_{k}} v_{k}(T, x(T))=v_{k}(T, x)=0 \quad$ by $\quad(16)$ $\mathbf{E}_{t_{k}, x_{k}} v_{k}(t, x(t))=\mathbf{E}_{t_{k}, x_{k}} v_{k}(t, x)=v_{k}(t, x)$. So,

$$
\mathbf{E}_{t_{k}, x_{k}}\left\{\int_{t}^{T} L v(s, x) \mathrm{d} s\right\}=-v_{k}(t, x) .
$$

and

Substituting this equality into (18), we get the statement of Lemma 2.

\section{Theorem 1. Let}

(1) Unique solution of problems (6)-(8) exists with finite second moment for each $t$.

(2) Sequence of functions $v_{k} \in V, k \geq 0$, and control $u_{k}^{0} \in U, k \geq 0$, which satisfy for all $t \in\left[t_{k}, T\right]$ in the equation

$$
L v_{k}(t, x)+G\left(t, x, u_{k}^{0}(t, x)\right)=0
$$

with boundary conditions

$$
v_{k}(T, x)=F(x(T)),
$$

exist.

(3) $\forall t \in[0, T], \forall u_{k} \in U, k \geq 0$, and the inequality hold:

$$
L v_{k}(t, x)+G\left(t, x, u_{k}(t, x)\right) \geq 0,
$$

where $L=L(t, x, u)$ is WIO (10) on solutions of (6)-(8).

Then, the control $u_{k}^{0}$ is optimal, and for $\forall t \in\left[t_{0}, T\right]$,

$$
I^{u_{k}^{0}}(t, x)=\inf _{u \in U} I^{u}(t, x)=v_{k}(t, x) .
$$

The sequence of functions $v_{k}(t, x)$ is called the cost, or the Bellman function, and equation (21) can be written as the Bellman equation:

$$
\inf _{u \in U}\left[L\left(t, x_{k}, u\right) v_{k}\left(t, x_{k}\right)+G\left(t, x_{k}, u\right)\right]=0
$$

Proof. The optimal control $u_{k}^{0} \in U$ is an admissible control. So, there is a solution $x\left(t_{k}, t_{0} y, h, u_{k}^{0}\right)$ for which (21) takes the form

$$
L v_{k}\left(t, x\left(t_{k}, t_{0}, y, h, u_{k}^{0}\right)\right)+G\left(t, x\left(t_{k}, t_{0}, y, h, u_{k}^{0}\right), u_{k}^{0}\right)=0
$$

$u_{k}^{0}$ is chosen at $\left(t, x\left(t, t_{0}, y, h, u_{k}^{0}\right)\right), t \in\left[t_{k}, t_{k+1}\right)$.

Let us integrate (26) from $t$ to $T$, obtain the expectation, and, taking into account (22), obtain

$$
v_{k}(t, x)=\mathbf{E}_{t_{k}, x_{k}}\left\{F(x(T))+\int_{t}^{T} L v_{k}\left(\tau, x\left(\tau, t_{0}, y, h, u_{k}^{0}\right)\right) \mathrm{d} \tau\right\} .
$$

Now, let $u_{k}=u_{k}(t)$ be any other admissible control from $U$. Then, by condition (3), the following inequality holds: for $\forall u_{k} \in U, k \geq 0$,

$$
L v\left(\tau, x\left(\tau, t_{0}, y, h, u\right)\right)+G\left(\tau, x\left(\tau, t_{0}, y, h, u\right), u(\tau)\right) \geq 0 .
$$

Let us integrate (28) with respect to $\tau \in\left[t_{k}, T\right]$ and obtain the expectation $\mathbf{E}$ with fixed $\tau$ and initial value $x$. Taking into account Lemmas 1 and 2 and boundary condition (22), we obtain

$$
\begin{aligned}
& v_{k}\left(t, x\left(t, t_{0}, y, h, u_{k}^{0}\right)\right) \\
& \leq \mathbf{E}_{t_{k}, x_{k}}\left\{F\left(x\left(T, u_{k}\right)\right)+\int_{t_{k}}^{T} G\left(\tau, x\left(\tau, t_{0}, y, h, u_{k}\right), u_{k}(\tau, x)\right) \mathrm{d} \tau\right\} \\
& =v_{k}\left(t, x\left(t, t_{0}, y, h, u_{k}\right)\right) .
\end{aligned}
$$

This is the definition of optimal control $u_{k}^{0}(t, x)$ in the sense of the minimization of the functional $I^{u}(t, x)$. The theorem is proved.

2.1. General Solution of the Problem of the Optimal Control. According to $[10,11,26]$, WIO $(10)$ has the form 


$$
\begin{aligned}
L v_{k}(t, x)= & \frac{\partial v_{k}(t, x)}{\partial t}+\left(\nabla v_{k}(t, x), a(t, y, x, u)\right)+\frac{1}{2} S p\left(b^{T}(t, y, x, u) \cdot \nabla^{2} v_{k}(t, x) \cdot b(t, y, x, u)\right) \\
& +\int_{R^{m}}\left[v_{k}(t, x+c(t, y, x, u, z))-v_{k}(t, x)-\left(\nabla v_{k}(t, x)\right)^{T} \cdot c(t, y, x, u, z)\right] \Pi(\mathrm{d} z) \\
& +\sum_{j \neq i}^{N}\left[\int_{R^{m}} v_{j}(t, x) p_{i j} t,(z / x) \mathrm{d} z-v_{i}(t, x)\right] q_{i j} .
\end{aligned}
$$

$(\cdot, \cdot)$ is a scalar product, $\left(\nabla v_{k}\right):=\left(\left(\partial v_{k} / \partial x_{1}\right), \ldots,\left(\partial v_{k} / \partial x_{m}\right)\right)^{T}, \quad\left(\nabla^{2} v_{k}\right):=\left[\partial^{2} v_{k} /\right.$ $\left.\partial x_{i} \partial x_{j}\right]_{i, j=1}^{m}, k \geq 0$; «" ${ }^{T}$ is the sign of transpose, $S p$ is the trace of a matrix, and $p_{i j} t,(z / x)$ is the conditional density of distribution

$$
\mathbf{P}\left\{x(\tau) \in \frac{[z, z+\mathrm{d} z]}{x(\tau-0)}=x\right\}=p_{i j}\left(\frac{\tau}{z / x}\right) \mathrm{d} z+o(\mathrm{~d} z) .
$$

$$
\begin{aligned}
& \frac{\partial v_{k}^{0}(t, x)}{\partial t}+\left(\frac{\partial v_{k}^{0}(t, x)}{\partial x}\right)^{T} \cdot a\left(t, y_{i}, x, u\right)+\frac{1}{2} S p\left(b^{T}\left(t, y_{i}, x, u\right) \cdot \frac{\partial^{2} v_{k}^{0}(t, x)}{\partial x^{2}} \cdot b\left(t, y_{i}, x, u\right)\right) \\
& \quad+\int_{\mathbf{R}^{m}}\left[v_{k}^{0}\left(t, x+c\left(t, y_{i}, x, u, z\right)\right)-v_{k}^{0}(t, x)-\left(\frac{\partial v_{k}^{0}(t, x)}{\partial x}\right)^{T} \cdot c\left(t, y_{i}, x, u, z\right)\right] \Pi(\mathrm{d} z) \\
& \quad+\sum_{j \neq i}^{N}\left[\int_{\mathbf{R}^{m}} v_{j}^{0}(t, x) p_{i j}(t, z / x) \mathrm{d} z-v_{i}^{0}(t, x)\right] q_{i j}+G(t, x, u)=0,
\end{aligned}
$$

with boundary condition

$$
v_{k}^{0}(T, x)=F(x(T)) .
$$

By assumption, $\xi(\tau-0)=y_{i}$ and $\xi(\tau)=y_{j}$.

The first equation for $v_{k}^{0}(t, x), k \geq 0$, can be obtained by substituting (30) in (21). Then, the required equation takes the form 
with Markov switching

$$
\left.\Delta x(t)\right|_{t=t_{k}}=g\left(t_{k}-, \xi\left(t_{k^{-}}\right), \eta_{k}, x\left(t_{k}-\right)\right),
$$

and initial conditions

$$
\begin{gathered}
x(0)=x_{0} \in \mathbf{R}^{m}, \\
\xi(0)=y \in \mathbf{Y}, \\
\eta_{0}=h \in \mathbf{H} .
\end{gathered}
$$

Here, $A, B, \sigma$, and $C$ are piecewise continuous integrable matrix functions of appropriate dimensions.

The optimal control problem for systems (35)-(37) is to find the control $u_{i k}^{0}, i \in\{1, \ldots N\}, k \geq 0$, from set $U$ of admissible controls such that it minimizes the functional of quality:

$$
I\left(u_{i k}\right):=I^{u_{i k}}(t, x):=\sum_{k=0}^{\bar{N}} \mathbf{E}_{t_{k}, x_{k}}\left\{x^{T}(T) M_{0}\left(\xi(t), \eta_{k}\right) x(T)+\int_{t}^{T}\left[u^{T}(s) M_{1}\left(s, \xi(s), \eta_{k}\right) u(s)+x^{T}(s) M_{2}\left(s, \xi(s), \eta_{k}\right) x(s)\right] \mathrm{d} s\right\}
$$

$M_{1}\left(t, \xi(t), \eta_{k}\right)$ is a uniformly positive definite $m \times m$-matrix with respect to $t \in[0, T]$, and $M_{0}\left(\xi(t), \eta_{k}\right)$ and $M_{2}\left(t, \xi(t), \eta_{k}\right)$ are nonnegative definite $m \times m$ matrices. To simplify, let us denote

$$
\begin{aligned}
A_{i}(t) & :=A\left(t, y_{i}\right), \\
B_{i}(t) & :=B\left(t, y_{i}\right), \\
\sigma_{i}(t) & :=\sigma\left(t, y_{i}\right), \\
C_{i}(t, z) & :=C\left(t, y_{i}, z\right), \\
M_{0 i k} & :=M_{0}\left(y_{i}, \eta_{k}\right), \\
M_{1 i k}(t) & :=M_{1}\left(t, y_{i}, \eta_{k}\right), \\
M_{2 i k}(t) & :=M_{2}\left(t, y_{i}, \eta_{k}\right) .
\end{aligned}
$$

Theorem 2. The optimal control for problems (35)-(38) has the form

$$
u_{i k}^{0}(t, x)=-M_{1 i k}^{-1}(t) B_{i}^{T}(t) P_{i k}(t) x(t),
$$

where the nonnegative definite $m \times m$ matrix $P_{i k}(t):=P\left(t, \xi(t), \eta_{k}\right)$ belongs to the Bellman functional:

$$
\begin{gathered}
v_{i k}^{0}(t, x)=x^{T}(t) P_{i k}(t) x(t), \\
v_{i k}^{0}(T, x)=x^{T}\left(t_{k}\right) M_{0 i k} x\left(t_{k}\right) .
\end{gathered}
$$

Proof. The Bellman equation for (35)-(37) has the form

$$
\inf _{u \in U}\left[L v_{i k}^{0}(t, x)+u_{i k}^{T}(t, x) M_{1 i k}(t) u_{i k}(t, x)+x^{T}(t) M_{2 i k}(t) x(t)\right]=0,
$$

where

$$
\begin{aligned}
L v_{i k}^{0}(t, x)= & \frac{\partial v_{i k}^{0}(t, x)}{\partial t}+\left[A_{i}(t) x(t)+B_{i}(t) u_{i k}(t, x)\right]^{T} \nabla v_{i k}^{0}(t, x) \\
& +\frac{1}{2} S p\left(\sigma_{i}^{T}(t) \nabla^{2} v_{i k}^{0}(t, x) \sigma_{i}(t)\right) \\
& +\int_{\mathbf{R}^{m}}\left[v_{i k}^{0}\left(t, x+C_{i}(t, z)\right)-v_{i k}^{0}(t, x)\right. \\
& \left.-\left(\frac{\partial v_{i k}^{0}(t, x)}{\partial x}\right)^{T} \cdot C_{i}(t, z)\right] \Pi(\mathrm{d} z) \\
& +\sum_{j \neq i}^{N}\left[v_{j k}^{0}(t, x)-v_{i k}^{0}(t, x)\right] q_{i j} \\
& +\int_{\mathbf{H}} v_{i k}^{0}\left(t, x+g\left(t_{k}-, \xi\left(t_{k}-\right), \eta_{k}, x\left(t_{k}-\right)\right)\right) \mathbf{P}_{k}(h, Z)-v_{i k}^{0}(t, x) .
\end{aligned}
$$

Substitute (43) into (42):

$$
\begin{aligned}
& \frac{\partial v_{i k}^{0}(t, x)}{\partial t}+\left[A_{i}(t) x(t)+B_{i}(t) u_{i k}(t, x)\right]^{T} \nabla v_{i k}^{0}(t, x) \\
& +\frac{1}{2} S p\left(\sigma_{i}^{T}(t) \nabla^{2} v_{i k}^{0}(t, x) \sigma_{i}(t)\right) \\
& +\int_{\mathbf{R}^{m}}\left[v_{i k}^{0}\left(t, x+C_{i}(t, z)\right)-v_{i k}^{0}(t, x)\right. \\
& \left.+\left(\frac{\partial v_{i k}^{0}(t, x)}{\partial x}\right)^{T} \cdot C_{i}(t, z)\right] \Pi(\mathrm{d} z) \\
& +\sum_{j \neq i}^{N}\left[v_{j k}^{0}(t, x)-v_{i k}^{0}(t, x)\right] q_{i j} \\
& +\int_{\mathbf{H}} v_{i k}^{0}\left(t, x+g\left(t_{k}-, \xi\left(t_{k}-\right), \eta_{k}, x\left(t_{k}-\right)\right)\right) \mathbf{P}_{k}(h, Z)-v_{i k}^{0}(t, x) \\
& +u_{i k}^{T}(t, x) M_{1 i k}(t) u_{i k}(t, x)+x^{T}(t) M_{2 i k}(t) x(t)=0 .
\end{aligned}
$$


The expression for the optimal control can be obtained by differentiating (44) because $u_{i k}(t, x)=u_{i k}^{0}(t, x)$ minimizes the left side of (44):

$$
u_{i k}^{0}(t, x)=-\frac{1}{2} M_{1 i k}^{-1}(t) B_{i}^{T}(t) \nabla v_{i k}^{0}(t, x),
$$

where

$$
\nabla v_{i k}^{0}(t, x)=2 P_{i k}(t) x(t)
$$

So,

$$
u_{i k}^{0}(t, x)=-M_{1 i k}^{-1}(t) B_{i}^{T}(t) P_{i k}(t) x(t) .
$$

The theorem is proved.

2.3. Construction of the Bellman Equation. Substituting (40) and (41) into (42), we obtain the following equation for $\forall t \in\left[t_{k}, t_{k+1}\right):$

$$
\begin{aligned}
x^{T}( & (t) \frac{\mathrm{d} P_{i k}(t)}{\mathrm{d} t} x(t)+2\left[A_{i}(t) x(t)\right. \\
& \left.-B_{i}(t) M_{1 i k}^{-1}(t) B_{i}^{T}(t) x(t)\right] P_{i k}(t) x(t)+S p\left(\sigma_{i}^{T}(t) P_{i k}(t) \sigma_{i}(t)\right) \\
& +\int_{\mathbf{R}^{m}} x^{T}(t) C_{i}(t, z) P_{i k}(t) C_{i}^{T}(t, z) x(t) \Pi(\mathrm{d} z) \\
& +\sum_{j \neq i}^{N}\left(x^{T}(t) P_{j k}(t) x(t)-x^{T}(t) P_{i k}(t) x(t)\right) q_{i j} \\
& +\left[M_{1 i k}^{-1}(t) B_{i}^{T}(t) P_{i k}(t) x(t)\right]^{T} \\
& M_{1 i k}(t) M_{1 i k}^{-1}(t) B_{i}^{T}(t) P_{i k}(t) x(t)+x^{T}(t) M_{2 i k}(t) x(t)=0 .
\end{aligned}
$$

Equating the quadratic form with respect to $x$ and expressions that do not depend on $x$ to zero and taking into account the matrix equality $2 x^{T} P_{i k} A_{i} x=x^{T}\left(P_{i k} A_{i}+A_{i}^{T} P_{i k}\right) x$, we obtain the system of differential equations for finding matrices $P_{i k}(t)$, $t \in\left[t_{k}, t_{k+1}\right), i \in\{1, \ldots N\}$, and $k \geq 0$ :

$$
\begin{gathered}
\frac{\mathrm{d} P_{i k}(t)}{\mathrm{d} t}+A_{i}^{T}(t) P_{i k}(t)+P_{i k}(t) A_{i}(t)-B_{i}(t) M_{1 i k}^{-1}(t) B_{i}^{T}(t) P_{i k}(t) \\
+\int_{\mathbf{R}^{m}} C_{i}(t, z) P_{i k}(t) C_{i}^{T}(t, z) \Pi(\mathrm{d} z) \\
+\sum_{j \neq i}^{N}\left(P_{j k}(t)-P_{i k}(t)\right) q_{i j} \\
+\left[M_{1 i k}^{-1}(t) B_{i}^{T}(t) P_{i k}(t)\right]^{T} B_{i}^{T}(t) P_{i k}(t)+M_{2 i k}(t)=0 \\
\quad S p\left(\sigma_{i}^{T}(t) P_{i k}(t) \sigma_{i}(t)\right)=0
\end{gathered}
$$

with boundary conditions

$$
P_{i k}(T)=M_{0 i k}
$$

Thus, the following theorem holds.
Theorem 3. If the control cost is found in the form (38) for systems (35)-(37), then the system of differential equations for finding the matrices $P_{i k}(t), t \in\left[t_{k}, t_{k+1}\right), i \in\{1, \ldots N\}$, and $k \geq 0$, has forms (49)-(51).

Furthermore, the problem of the existence of solutions of problems (49)-(51) must be solved. Let us use the Bellman method of iterations [18]. For simplicity, consider the interval $\left[t_{k}, t_{k+1}\right)$, where $\xi(t)=y_{i}$, and omit all indices «ik» in $u, v$, and $P$. First, we define the zero approximation:

$$
u_{0}(t, x)=-M_{1}^{-1}(t) B^{T}(t) P_{0}(t) x(t),
$$

where $P_{0}(t) \geq 0$ is the bounded and piecewise-continuous matrix. Let us substitute (52) into (34) and calculate the value of $v_{1}(t, x)$ for the resulting equation, which corresponds to the control (42).

Then, substituting $v_{1}(t, x)$ into Bellman equation (42), the control $u_{1}(t, x)$ which minimizes (42) will be found. Continuing this process, one can construct a sequence of controls $u_{n}(t, x)$ and functionals $v_{n}(t, x)$ of the following form:

$$
\begin{aligned}
u_{n}(t, x) & =-M_{1}^{-1}(t) B^{T}(t) P_{n}(t) x(t), \\
v_{n}(t, x) & =x^{T}(t) P_{n}(t) x(t), \\
v_{n}(T, x) & =x^{T}\left(t_{k}\right) M_{0} x\left(t_{k}\right),
\end{aligned}
$$

where $P_{n}(t), t \in\left[t_{k}, t_{k+1}\right)$ is the solution of boundary value problems (49)-(51) for $T:=t_{k+1}$.

The following estimate is valid for $\forall n \geq 1$ :

$$
v_{n-1}(t, x) \geq v_{n}(t, x) \geq 0, \quad \forall t \in\left[t_{k}, t_{k+1}\right) \text {. }
$$

The convergence of functionals $v_{n}(t, x)$ to $v^{0}(t, x)$, the convergence of controls $u_{n}(t, x)$ to $u^{0}(t, x)$, and the convergence of a sequence of matrices $P_{n}(t)$ to $P(t)$ can be proved by using (54) $[19,20]$.

The following estimate is valid:

$$
\max _{t \in\left[t_{k}, t_{k+1}\right)}\left\|P(t)-P_{n}(t)\right\| \leq \frac{C}{n !}, \quad C<\infty, k \geq 1 .
$$

Thus, the following theorem holds.

Theorem 4. An approximate solution of the problem of synthesis of the optimal control for (35)-(38) is found using the Bellman successive approximation method for which the $n$-th approximation of the control and the Bellman functional for each interval $\left[t_{k}, t_{k+1}\right), k \geq 0$, are found from formula (53), and the error is estimated by inequality (55).

2.4. Model Example. Let us consider the scalar case with two states for Markov chains $\xi$ and $\eta: \xi=[1,2]$ and $\eta=\left[\eta_{1}, \eta_{2}\right]$. Define the following initial data for system (35):

(1) $\xi=1, \quad A_{1}(t)=-1, \quad B_{1}(t)=1, \quad \sigma_{1}(t)=0.1, \quad$ and $C_{1}(t, z)=z^{2}$

(2) $\xi=2, A_{2}(t)=-2.25, \quad B_{2}(t)=1, \quad \sigma_{2}(t)=0.2$, and $C_{2}(t, z)=z^{3}$ 


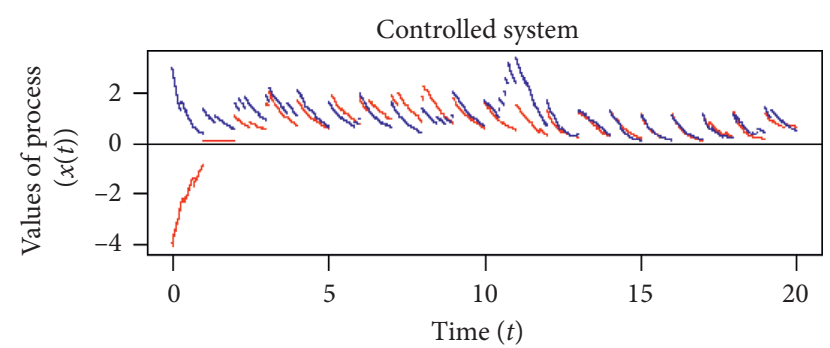

FIgURE 1: Two solutions of systems (35)-(37) with initial conditions -4 and 3 and parameters from the model example.

Generator of the Markov process $\xi$ is defined as $Q=\left(\begin{array}{cc}-0.5 & 0.5 \\ 0.5 & -0.5\end{array}\right)$, and the transaction matrix for the Markov chain $\eta$ is $\mathbf{P}=\left(\begin{array}{ll}0.3 & 0.7 \\ 0.8 & 0.2\end{array}\right)$.

Then, define $M_{0}, M_{1}$, and $M_{2}$ for (38):

$$
\begin{aligned}
& M_{011}=M_{021}=M_{012}=M_{022}=8, \\
& M_{111}=0.7, \\
& M_{112}=0.8, \\
& M_{121}=0.9, \\
& M_{122}=0.4, \\
& M_{211}=0.5, \\
& M_{212}=0.4, \\
& M_{221}=1, \\
& M_{222}=0.9 .
\end{aligned}
$$

The Bellman functional considers the following form: $v_{i k}^{0}(t, x)=P_{i k} x^{2}(t), i, k \in\{1,2\}$.

System (49) for finding $P_{i k}, i, k \in\{1,2\}$, takes the form

$$
\begin{aligned}
& 2 A_{i} P_{i k}-B_{i}^{2} M_{1 i k}^{-1} P_{i k}+\int_{\mathbf{R}^{1}} C_{i}^{2}(z) P_{i k} \Pi(\mathrm{d} z) \\
& \quad+\sum_{j \neq i}^{2}\left(P_{j k}-P_{i k}\right) q_{i j}+M_{1 i k}^{-1} B_{i}^{2} P_{i k}^{2}+M_{2 i k}=0, \quad i, k \in\{1,2\} .
\end{aligned}
$$

Substituting values of $A, B, M_{0}, M_{1}$, and $M_{2}$, we obtain

$$
\begin{aligned}
& P_{11}=0.1194, \\
& P_{12}=0.1004, \\
& P_{21}=0.1504, \\
& P_{22}=0.1122 .
\end{aligned}
$$

So, the optimal control for our case has a form

$$
u^{0}(t, x)= \begin{cases}-0.08358 x(t), & \xi=1, \eta=\eta_{1}, \\ -0.08032 x(t), & \xi=1, \eta=\eta_{2}, \\ -0.13536 x(t), & \xi=2, \eta=\eta_{1}, \\ -0.04488 x(t), & \xi=2, \eta=\eta_{2} .\end{cases}
$$

The results of simulation of two trajectories of the random process $x(t)$ are shown in Figure 1. This figure shows two trajectories of solutions with a positive (blue line) and negative (red line) initial condition $x_{0}$. As we can see from this figure, the solutions are stabilized because the optimal control satisfies the conditions of Theorem 1 with the quadratic functional.

\section{Conclusions}

In this paper, the problem of synthesis of the optimal control of stochastic dynamical systems of a random structure with Poisson perturbations that are under the influence of pulse switching of the Markov chain type is solved. In the linear case, an algorithm for finding the optimal control is obtained, and its convergence is justified.

\section{Data Availability}

No data were used to support this study.

\section{Conflicts of Interest}

The authors declare that there are no conflicts of interest regarding the publication of this article.

\section{References}

[1] V. S. Korolyuk and V. V. Korolyuk, Stochastic Models of Systems, Kluwer, Boston, MA, USA, 1999.

[2] F. Long, H. Huang, and A. Ding, "Stochastic stabilization of Ito stochastic systems with Markov jumping and linear fractional uncertainty," Journal of Control Science and Engineering, vol. 2013, Article ID 697849, 14 pages, 2013.

[3] M. Bujorianu and J. Lygeros, "Toward a general theory of stochastic hybrid systems," Lecture Notes in Control and Information Sciences (LNCIS), vol. 337, pp. 3-30, Springer, Berlin, Germany, 2006.

[4] L. Hu, P. Shi, and B. Huang, "Stochastic stability and robust control for sampled-data systems with Markovian jump parameters," Journal of Mathematical Analysis and Applications, vol. 313, no. 2, pp. 504-517, 2006.

[5] M. Aliyu and E. K. Boukas, "Robust $H_{\infty}$ control for Markovian jump nonlinear systems," IMA Journal of Mathematical Control and Information, vol. 17, no. 3, pp. 295-308, 2000.

[6] V. Dragan and T. Morozan, "The linear quadratic optimization problems for a class of linear stochastic systems with multiplicative white noise and Markovian jumping," IEEE Translation on Automatic Control, vol. 49, no. 5, pp. 665-675, 2004.

[7] K. Benjelloun, E. K. Boukas, and O. L. V. Costa, " $H_{\infty}$-control for linear time-delay systems with markovian jumping parameters," Journal of Optimization Theory and Applications, vol. 105, no. 1, pp. 73-95, 2000.

[8] I. Y. Katz, The Method of Lyapunov Functions in Problems of Stability and Stabilization of Systems of Random Strucure, UGAPS, Ekaterinburg, Russia, 1998.

[9] M. L. Sverdan and Y. F. Tsarkov, Stability of Stochastic Impulse Systems, RTU, Riga, Latvia, 1994.

[10] T. O. Lukashiv, I. V. Yurchenko, and V. K. Yasinskii, "Lyapunov function method for investigation of stability of stochastic Ito random-structure systems with impulse Markov switchings. I. General theorems on the stability of stochastic 
impulse systems," Cybernetics and Systems Analysis, vol. 45, no. 2, pp. 281-290, 2009.

[11] T. O. Lukashiv, I. V. Yurchenko, and V. K. Yasinsky, "Lyapunov function method for investigation of stability of stochastic Ito random-structure systems with impulse Markov switchings. II. First-approximation stability of stochastic impulse systems with markov parameters," Cybernetics and Systems Analysis, vol. 45, no. 3, pp. 464-476, 2009.

[12] T. O. Lukashiv, V. K. Yasinskiy, and E. V. Yasinskiy, "Stabilization of stochastic diffusive dynamical systems with impulse markov switchings and parameters. Part I. Stability of impulse stochastic systems with markov parameters," Journal of Automation and Information Sciences, vol. 41, no. 2, pp. 1-24, 2009.

[13] T. O. Lukashiv, L. I. Yasinskaya, and V. K. Yasinskiy, "Stabilization of stochastic diffusive dynamical systems with impulse markov switchings and parameters. Part II. Stabilization of dynamical systems of random structure with external markov switchings," Journal of Automation and Information Sciences, vol. 41, no. 4, pp. 26-42, 2009.

[14] T. Lukashiv and I. Malyk, "Existence and uniqueness of solution of stochastic dynamic systems with Markov switching and concentration points," International Journal of Differential Equations, vol. 2017, Article ID 7958398, 5 pages, 2017.

[15] Y. F. Tsarkov, V. K. Yasinsky, and I. V. Malyk, "Stability in impulsive systems with Markov perturbations in averaging scheme. I. Averaging principle for impulsive Markov systems," Cybernetics and Systems Analysis, vol. 46, no. 6, pp. 975-985, 2010.

[16] Y. F. Tsarkov, V. K. Yasinsky, and I. V. Malyk, "Stability in impulsive systems with Markov perturbations in averaging scheme. 3. Weak convergence of solutions," Cybernetics and Systems Analysis, vol. 47, no. 3, pp. 442-458, 2011.

[17] I. I. Gikhman and A. V. Skorokhod, Controlled Stochastic Processes, Springer Verlag, New York, NY, USA, 1979.

[18] R. Bellman, Dynamic Programming, Princeton University Press, Princeton, NJ, USA, 1972.

[19] E. A. Andreeva, V. B. Kolmanovskii, and L. E. Shaikhet, Control of Hereditary Systems, Nauka, Moskow, Russia, 1992.

[20] T. O. Lukashiv, L. I. Yasinskaya, and V. K. Yasinskiy, "Synthesis of the optimal control for linear stochastic dynamical systems with finite aftereffect and Poisson disturbances," Journal of Automation and Information Sciences, vol. 40, no. 10, pp. 22-37, 2008.

[21] T. O. Lukashiv and I. V. Malyk, "Sufficient optimality conditions for stochastic dynamical systems of random structure with markovian switchings," Journal of Automation and Information Sciences, vol. 48, no. 6, pp. 60-67, 2016.

[22] A. Das, T. O. Lukashiv, and I. V. Malyk, "Optimal control synthesis for stochastic dynamical systems of random structure with the markovian switchings," Journal of Automation and Information Sciences, vol. 4, no. 49, pp. 37-47, 2017.

[23] I. V. Malyk, "Compensating operator and weak convergence of semi-Markov process to the diffusion process without balance condition," Journal of Applied Mathematics, vol. 2015, Article ID 563060, 7 pages, 2015.

[24] E. B. Dynkin, Markov Processes, Academic Press, New York, NY, USA, 1965.

[25] B. Oksendal, Stochastic Differential Equations, Springer, New York, NY, USA, 2013.

[26] T. Lukashiv, "One form of lyapunov operator for stochastic dynamic system with Markov parameters," Journal of Mathematics, vol. 2016, Article ID 1694935, 5 pages, 2016. 